\title{
Different Effects of Typical and Atypical Antipsychotics on Grey Matter in First Episode Psychosis: the ÆSOP Study
}

\author{
Paola Dazzan*,', Kevin D Morgan', Ken Orr², Gerard Hutchinson ${ }^{3}$, Xavier Chitnis $^{4}$, John Suckling ${ }^{5}$, \\ Paul Fearon', Philip K McGuire', Rosemarie M Mallett ${ }^{6}$, Peter B Jones ${ }^{5}$, Julian Leff ${ }^{6}$ and Robin M Murray' \\ 'Division of Psychological Medicine, Institute of Psychiatry, King's College London, UK; ${ }^{2}$ Sir Charles Gairdner Hospital, Department of Psychiatry, \\ Perth, Australia; ${ }^{3}$ Department of Psychiatry, University of West Indies, Trinidad, Trinidad and Tobago; ${ }^{4}$ Department of Neurology, Institute of \\ Psychiatry, King's College London, UK; ${ }^{5}$ Addenbrooke's Hospital, Department of Psychiatry, University of Cambridge, Cambridge, UK; ${ }^{6}$ Institute of \\ Psychiatry, Section of Social Psychiatry, King's College London, UK
}

\begin{abstract}
Typical antipsychotic drugs act on the dopaminergic system, blocking the dopamine type 2 (D2) receptors. Atypical antipsychotics have lower affinity and occupancy for the dopaminergic receptors, and a high degree of occupancy of the serotoninergic receptors 5-HT2A. Whether these different pharmacological actions produce different effects on brain structure remains unclear. We explored the effects of different types of antipsychotic treatment on brain structure in an epidemiologically based, nonrandomized sample of patients at the first psychotic episode. Subjects were recruited as part of a large epidemiological study (ÆSOP: aetiology and ethnicity in schizophrenia and other psychoses). We evaluated 22 drug-free patients, 32 on treatment with typical antipsychotics and 30 with atypical antipsychotics. We used high-resolution MRI and voxel-based methods of image analysis. The MRI analysis suggested that both typical and atypical antipsychotics are associated with brain changes. However, typicals seem to affect more extensively the basal ganglia (enlargement of the putamen) and cortical areas (reductions of lobulus paracentralis, anterior cingulate gyrus, superior and medial frontal gyri, superior and middle temporal gyri, insula, and precuneus), while atypical antipsychotics seem particularly associated with enlargement of the thalami. These changes are likely to reflect the effect of antipsychotics on the brain, as there were no differences in duration of illness, total symptoms scores, and length of treatment among the groups. In conclusion, we would like to suggest that even after short-term treatment, typical and atypical antipsychotics may affect brain structure differently.

Neuropsychopharmacology (2005) 30, 765-774, advance online publication, 9 February 2005; doi: I 0. I038/sj.npp. I 300603
\end{abstract}

Keywords: schizophrenia; psychosis; antipsychotics; MRI; basal ganglia; frontal lobe

\section{INTRODUCTION}

The exact mechanism by which typical and atypical antipsychotics exert their different therapeutic effects is still unclear (Meltzer et al, 1999). The introduction of antipsychotic drugs in the early 1950s revolutionized the treatment of psychoses, and schizophrenia in particular. These drugs (typical antipsychotics, for example, haloperidol and chlorpromazine) act on the dopaminergic system, blocking the dopamine type 2 (D2) receptors in mesolimbic areas (Carlsson, 1978). Owing to this D2 blockade, they also induce a number of side effects, among which extrapyramidal symptoms are the most prominent (Dazzan and

\footnotetext{
*Correspondence: P Dazzan, Division of Psychological Medicine, Institute of Psychiatry, Box 63, De Crespigny Park, London SE5 8AF, UK, Tel: + 44207848 0590, Fax: + 44207 70। 9044,

E-mail: spcbpad@iop.kcl.ac.uk

Received 20 February 2004; revised 23 September 2004; accepted 28 September 2004

Online publication: 30 September 2004 at http://www.acnp.org/citations/ NPP09300404008 I/default.pdf
}

Murray, 2002; Dazzan et al, 2004). Recently, a new generation of antipsychotic drugs, the atypical antipsychotics, have become available (for example, clozapine, risperidone, olanzapine, and quetiapine). Atypical antipsychotics have lower affinity and occupancy for the dopaminergic receptors, and a high degree of occupancy of the serotoninergic receptors 5-HT2A (Meltzer et al, 1989). Compared to typical antipsychotics, atypicals induce fewer extra-pyramidal side effects, but the exact neurobiological substrate of this difference is still unknown.

One way to better understand the mechanisms underlying the different side effects of typical and atypical antipsychotics is to use neuroimaging to investigate brain changes associated with a specific class of antipsychotic drug. Until now, structural magnetic resonance imaging (MRI) studies of antipsychotics effects have been restricted to the evaluation of a few selected subcortical regions. The most consistent finding has been that typical antipsychotics are associated with an enlargement of the basal ganglia, and that this enlargement does not occur, or can be reversed, with atypical antipsychotics (Chakos et al, 1994; Corson 
et al, 1999). Different effects of typicals and atypicals have also been described at cortical level in functional imaging studies. Specifically, typical antipsychotic agents seem to significantly reduce relative blood flow in the frontal cortex, while atypicals may be associated with a smaller blood flow decrease in this area (Honey et al, 1999; Miller et al, 2001).

One limitation of the existing studies on effects of antipsychotics on brain structure is that they have often investigated patients treated with antipsychotics for many years, with different types of antipsychotic drugs, and at a variety of doses. This makes it difficult to disentangle which brain changes are due to a specific class of antipsychotics, and which are due to the illness and its progression (Dazzan and Murray, 1999). Therefore, these questions can be better addressed by investigating subjects at the initial stages of psychosis, when treatment would have occurred for only a short time, and the dose taken is easier to establish. They therefore represent an ideal sample to investigate the differential, short-term, effects of antipsychotics on brain structure.

In the present exploratory study, we investigated the relationship between brain structure and antipsychotic treatment in an epidemiologically based sample of patients at the first psychotic episode, both antipsychotic-treated and antipsychotic-free. Using an epidemiologically based sample limits the potential bias of recruiting subjects selected because of their treatment. We collected a complete medication history for each subject, and used highresolution MRI and voxel-based methods of image analysis. Voxel-based analysis has the major advantage of allowing the evaluation of the entire brain rather than of a few preselected regions; furthermore, it is automated, which means that it does not require the identification of anatomical boundaries nor manual tracing of the regions of interest (Dazzan et al, 2004). This study aimed: (1) to investigate whether antipsychotics have a measurable effect on brain anatomy; and if so, (2) to investigate if typical and atypical antipsychotics affect brain anatomy differently.

\section{PATIENTS AND METHODS}

Subjects were recruited as part of a large epidemiological study (ÆSOP: aetiology and ethnicity in schizophrenia and other psychoses), carried out in three English cities, which investigated the higher rates of schizophrenia in the African-Caribbean population in the United Kingdom (Dazzan et al, 2004). Ethical approval for the study was granted by the Ethical Committee of the Institute of Psychiatry, and the participants gave written informed consent, in accordance with the Declaration of Helsinki.

As part of the South London arm of this study, we approached subjects aged 16-65, who consecutively presented for the first time to the local psychiatric services for a functional psychotic illness (ICD10 F 10-19, excluding coding F 1 x.0 for acute intoxication; F 20-29 and F 30-39, psychotic codings) (World Health Organisation, 1992), over a 3-year period. Exclusion criteria were: (a) a history of head trauma resulting in loss of consciousness for over $1 \mathrm{~h}$; (b) the presence of a disease of the central nervous system; (c) moderate or severe learning disabilities as defined by ICD-10 (World Health Organisation, 1992); (d) poor fluency in English language; (e) transient psychotic symptoms resulting from acute intoxication as defined by ICD-10 (World Health Organisation, 1992), following the administration of alcohol or other psychoactive substance.

A total of 281 patients met the inclusion criteria and were invited to participate in the overall London arm of the ÆSOP study: 90 refused to take part in the investigation. Of the remaining 191 who participated, 115 patients consented to have an MRI scan. These 115 patients were on average 6 years younger (mean age $27.9 \pm 8.4$ years vs $33.7 \pm 12.3$ years, $t=3.5, P=0.001$ ) and had a higher proportion of white British subjects ( 36 vs $18 \%, \chi^{2}=6.95, P=0.008$ ). They were comparable to the total sample in terms of gender. Ten patients terminated the scanning session before full image acquisition had been achieved and a further 15 scans were excluded from the analysis ( 13 due to subject motion, one because of congenital hydrocephalus, and one because of the presence of a subarachnoid cyst).

\section{Clinical Measures}

We interviewed patients using the WHO Schedules for Clinical Assessment in Neuropsychiatry (SCAN) (World Health Organisation, 1994). We made a diagnosis according to ICD-10 criteria (World Health Organisation, 1992) by consensus in meetings with senior clinicians (RM or JL) from the Institute of Psychiatry, in which all clinical information was presented. A total symptomatology score was obtained by summing the SCAN's individual symptom item scores as per Wing and Sturt (1978) procedure for the Present State Examination (PSE) (Wing et al, 1974; Wing and Sturt, 1978). This was an appropriate model to adopt as the SCAN incorporates the 10th edition of the PSE. Duration of illness (DOI) was operationalized as the time in weeks between the onset of psychotic symptoms and the MRI scan date. We used both the patients' medical notes and the information obtained from the SCAN interviews to establish the onset.

The premorbid IQ was estimated by the National Adult Reading Test (NART) (Nelson and Willison, 1991). We assessed handedness according to the Annett Hand Preference Questionnaire (Annett, 1970).

\section{Pharmacological Treatment}

From clinical notes, we completed a medication record for each patient. We calculated the total duration of antipsychotic exposure in days and the daily antipsychotic dose at the time of MRI scan, converted into chlorpromazine equivalents for typical antipsychotics (Bazire, 1998; Bezchlibnyk-Butler and Jeffries, 2000; Taylor et al, 1999). We also recorded information on treatment with anticholinergic drugs, antidepressants, and mood stabilizers. Therapeutic interventions (type of medication and length of treatment) were decided by the responsible clinical team, based on clinical presentation, and were not influenced by participation to the study. For the purposes of the main study, we obtained one MRI scan as soon as possible after first presentation to the services, independently on length of antipsychotic treatment. Depending on their current treatment, subjects were divided into three groups: (1) typical antipsychotics; (2) atypical antipsychotics; (3) drug-free. On the basis of existing literature on antipsychotic washout, we 
considered 'drug-free' those subjects who had not taken any antipsychotic in the 3 weeks prior to the MRI scan (Farde et al, 1986; Miller et al, 1997a,b, 2001). We considered subjects as being on treatment with typical antipsychotics if they had been taking one typical antipsychotic only for at least 2 weeks prior to MRI and had not taken more than one dose of an atypical antipsychotic during this time. The same criteria were used for the subjects on atypicals. Therefore, the allocation of subjects to each of these three groups was nonrandomized, but based on the medication prescribed by the in-charge clinician at the time of MRI scan. According to the existing literature on brain changes following administration of antipsychotics (Chakos et al, 1994; Cohen et al, 2003; Christensen et al, 2004; Grunder et al, 2003; Honey et al, 1999; Huang et al, 1999; Miller et al, 1997a, b, 2001; Wotanis et al, 2003), the mean length of treatment of 8 weeks of our sample would be sufficient to observe brain changes in association with antipsychotic use.

\section{Structural MRI}

Image acquisition. Scans were acquired with a GE Signa 1.5-T system (GE Medical Systems, Milwaukee), at the Maudsley Hospital, London. Contiguous, interleaved proton-density- and T2-weighted images, each 3-mm thick, were acquired in the coronal plane, to provide whole brain coverage. A repetition time (TR) of $4000 \mathrm{~ms}$ and effective echo times (TE) of 20 and $85 \mathrm{~ms}$ were used with an 8-echo train length. The matrix size was $256 \times 192$, collected from a rectangular field-of-view of $22 \times 16.5 \mathrm{~cm}^{2}$, giving an inplane resolution of $0.859 \mathrm{~mm}$ in both directions. The total acquisition time was $10 \mathrm{~min}$ and $12 \mathrm{~s}$.

Image processing. The methods used for segmentation and registration of each fast spin echo data set have been described in detail elsewhere (Bullmore et al, 1999; Suckling et al, 1999a). Briefly, extra-cerebral tissues were initially removed, using an automated algorithm. Manual editing of the skull-stripped images was necessary only to remove brainstem and cerebellum from the cerebral hemispheres and diencephalon. The probability of each intracerebral voxel belonging to each of four possible tissue classes (grey matter, white matter, cerebrospinal fluid (CSF), or dura/ vasculature) was then estimated with a modified fuzzy clustering algorithm (Suckling et al, 1999b). This type of segmentation assigns, to each voxel, a value in the range $0-1$ assuming to indicate the fraction of the voxel comprised by each tissue type (for example, a grey matter value of 0.7 , means that $70 \%$ of the tissue represented by that voxel is grey matter; therefore, the value indicates the proportion of the voxel occupied by grey matter).

A template image in the standard space of Talairach and Tournoux (1988) was constructed using the AFNI program from proton-density images acquired from six healthy subjects (Dazzan et al, 2004). Maps of tissue distribution were then registered onto the template by registering each proton density image using a nineparameter affine registration, minimizing the grey-level difference between images. This registration aligns all the images together, and scales them to the same gross dimensions. The derived mapping was then applied to the corresponding tissue maps.
Between-group differences in grey matter volume were estimated by fitting an analysis of covariance (ANCOVA) model at each intracerebral voxel in standard space covarying for age at scan and total grey matter volume (an estimate automatically provided by the program). Covarying for global grey matter may not be preferable to covarying for intracranial volume in a classical comparison of patients with schizophrenia $v s$ healthy controls, because there is often a reduction in cortical volume in patients with schizophrenia. In that case, covarying for this variable may therefore remove effects of interest. However, in the present study (consisting only of patient groups) there were no differences in total grey matter volume across the groups. Hence, we consider grey matter volume an appropriate covariate.

Permutation testing was used to assess statistical significance, and regional relationships were tested at the level of voxel clusters (Bullmore et al, 1999; Sigmundsson et al, 2001). Given that structural brain changes are likely to extend over a number of contiguous voxels, test statistics which incorporate spatial information, such as 3D cluster mass (the sum of suprathreshold voxel statistics), are generally more powerful than other test statistics, which are informed only by data at a single voxel. For each analysis, we calculate the critical value in the null distribution of cluster mass at which $<1$ cluster would be observed (expected number of false positive clusters ( $P$-value times number of tests) was $<1$ false positive), and the observed distribution is then thresholded at the corresponding $P$ value. We do not calculate a $P$-value for every cluster, but instead state that all our clusters are significant at a particular level (or less). We have already used this method successfully to investigate brain structure in this sample (Dazzan et al, 2004).

\section{RESULTS}

At the time of scan, 32 patients were on treatment with typical antipsychotics, 30 with atypical antipsychotics, six were receiving both typicals and atypicals, and 22 patients were drug-free (13 neuroleptic-naïve) (Table 1). We excluded the six patients on treatment with both typical and atypical antipsychotics from further analyses. Sociodemographic and clinical characteristics of the three groups are shown in Table 1. Patients on typicals were receiving: chlorpromazine, sulpiride, haloperidol, thioridazine, droperidol, trifluoperazine, zuclopenthixol (mean chlorpromazine equivalents: $269.5 \pm 245 \mathrm{mg}$ ). Patients on atypicals were receiving: olanzapine, risperidone, quetiapine, sertindole, amisulpiride. The number of subjects taking each of these atypical antipsychotic and the mean dose are shown in Table 1. Some patients were also receiving adjunctive anticholinergic treatment: $28 \%$ of those on typicals and $18 \%$ of those on atypicals. Some patients were also receiving antidepressants and a small minority was receiving mood stabilizers (Table 1).

There were no differences between the groups in age, gender, employment, and involvement in a relationship. Subjects on typicals and atypicals had less years of education than the drug-free group (F 6.8, $P=0.002$ ), and subjects on atypicals had a premorbid IQ significantly lower 
Table I Demographic and Clinical Characteristics of the Groups

\begin{tabular}{|c|c|c|c|c|}
\hline Characteristic & Typical $N=32$ & Atypical $\mathbf{N}=\mathbf{3 0}$ & Drug free $N=22$ & $\mathbf{P}$ \\
\hline Sex (\% female) & 38 & 30 & 50 & NS \\
\hline Age (years) & $28.4 \pm 7.8$ & $25 \pm 8$ & $28.2 \pm 7.8$ & NS \\
\hline Education (mean years) & $12.3 \pm 2.1$ & $12.3 \pm 1.8$ & $14.4 \pm 3$ & $0.002^{\mathrm{a}}$ \\
\hline Currently employed (\%) & 48 & 52 & 54 & NS \\
\hline Involvement in a relationship (\%) & 36 & 37 & 43 & NS \\
\hline \multicolumn{5}{|l|}{ Diagnosis (\%) } \\
\hline Schizophrenia (\% schizophreniform) & $47(19 \%)$ & $80(17 \%)$ & $32(18 \%)$ & $0.001^{b}$ \\
\hline Affective psychosis & 47 & 7 & 41 & \\
\hline Other psychosis (\% drug induced) & $6(0 \%)$ & $13(0.3 \%)$ & $27(0 \%)$ & \\
\hline Current typical antipsychotic dose (chlorpromazine equivalents in mg) & $269.5 \pm 245$ & - & - & - \\
\hline \multicolumn{5}{|c|}{ Current atypical antipsychotic dose ( $N$ of subjects taking the drug; mean dose in mg) } \\
\hline Olanzapine & - & $21(14)$ & - & - \\
\hline Risperidone & - & $5(4)$ & - & - \\
\hline Quetiapine & - & $2(400)$ & - & - \\
\hline Sertindole & - & I ( 16$)$ & - & - \\
\hline Amisulpiride & - & I (400) & - & - \\
\hline Currently taking anticholinergics (\%) & 28 & 18 & 0 & $0.02^{e}$ \\
\hline \multicolumn{5}{|l|}{ SCAN symptoms } \\
\hline Hypomania symptoms & $3.9 \pm 8$ & $1.8 \pm 3$ & $3.5 \pm 5$ & NS \\
\hline Negative symptoms & $0.4 \pm 1$ & $0.3 \pm 1$ & $0.1 \pm 0.2$ & NS \\
\hline Total symptoms score & $34.5 \pm 19$ & $33.4 \pm 16$ & $24.6 \pm 15$ & NS \\
\hline Gray matter $\left(\mathrm{cm}^{3}\right)$ & $584.5 \pm 58$ & $573.5 \pm 64$ & $568.9 \pm 64$ & NS \\
\hline
\end{tabular}

NS: nonsignificant

${ }^{a}$ One-way ANOVA: post hoc analyses showed that this was due to the drug-free group having more years of education than both the typical and atypical groups. ${ }^{\mathrm{b}} \chi^{2}$ : pairwise analysis showed that this was due to the atypical group having a significantly higher percentage of subjects with schizophrenia and a lower percentage of subjects with affective psychosis than the other two groups.

'One-way ANOVA: post hoc analyses showed that this was due to the atypical group having lower IQ than both the typical and the drug-free groups.

${ }^{\mathrm{d}}$ This refers to the comparison among the typical, atypical, and previously exposed groups.

${ }^{e} \chi^{2}$ : pairwise analysis showed that this was due to the drug-free group having a significantly lower percentage of subjects taking anticholinergic drugs than the other groups.

than both the typical and drug-free subjects (F 7.4, $P=0.001$ ). The group on atypicals had more subjects with a diagnosis of schizophrenia and less subjects with a diagnosis of affective psychosis than the other two groups $\left(\chi^{2} 13.2, P=0.001\right)$. There were no significant differences in DOI, total symptoms, and individual symptom domains among the three groups. Furthermore, there were no significant differences in length of treatment among the groups.

\section{Total Tissue Volumes}

There were no significant differences among the groups for total grey matter volume (Table 1). 


\section{Regional Differences}

Subjects on typical antipsychotics in comparison with drug-free subjects. In comparison with drug-free subjects, subjects taking typical antipsychotics had one cluster of grey matter excess and three clusters of grey matter volume reductions $(P \leqslant 0.002)$. The excess cluster was localized on the right lenticular nucleus (Table 2, Figure 1a). Of the three clusters of grey matter volume reductions (Table 2, Figure 1a), one cluster was on right insula, extending into inferior frontal (Brodmann's Area (BA) 47) and superior temporal (BA 22) gyri; a second cluster of grey matter reduction was centered on the left paracentral lobule (BAs 4, $5)$, and extended bilaterally and into the superior and medial frontal gyri (BAs 6, 31), and cingulate gyrus (BA 24); finally, a third cluster of grey matter reduction was centered on the left precuneus (BA 7). The three deficit clusters were negatively correlated with the current dose of antipsychotic (Pearson $r$ ranging from -0.45 to $0.50 ; P \leqslant 0.001$ ), while the lenticular nucleus excess was positively correlated with this dose (Pearson $r=0.29 ; P=0.01$ ).

Subjects on atypical antipsychotics in comparison with drug-free subjects. In comparison with drug-free subjects, subjects taking atypical antipsychotics showed a cluster of grey matter excess localized on the left and right thalami $(P=0.002)$ (Table 2, Figure 1b). The thalamus excess was positively correlated with the current dose of olanzapine (Pearson $r=0.31 ; \quad P=0.04$ ) (because of the lack of agreement on equivalents doses for atypical antipsychotics,

Table 2 Regional Differences in Grey Matter in Drug-Free Subjects, Subjects on Typicals, and Subjects on Typicals

\begin{tabular}{|c|c|c|}
\hline Anatomical area & $\begin{array}{l}\text { Number } \\
\text { of } \\
\text { voxels in } \\
\text { cluster }\end{array}$ & $\begin{array}{l}\text { Location of } \\
\text { cluster center } \\
x, y, z\end{array}$ \\
\hline \multicolumn{3}{|l|}{ Typicals vs drug free } \\
\hline \multicolumn{3}{|l|}{ Grey matter excesses in typicals } \\
\hline (I) R lenticular nucleus & 563 & $23.1,-3.1,2.6$ \\
\hline \multicolumn{3}{|l|}{ Grey matter deficits in typicals } \\
\hline $\begin{array}{l}\text { ( } 1) R \text { insula, extending into inferior } \\
\text { frontal gyrus (BA 47), superior } \\
\text { temporal gyrus (BA 22) }\end{array}$ & 741 & $37,12.3,-4.6$ \\
\hline $\begin{array}{l}\text { (2) } L \text { and } R \text { paracentral lobule (BA } \\
4,5 \text { ), extending into superior and } \\
\text { medial frontal gyri (BA 6, 3I), } \\
\text { cingulate gyrus (BA24) }\end{array}$ & 717 & $-0.0,-22.2,46.7$ \\
\hline (3) $L$ precuneus (BA 7) & 472 & $-1.3,-47.4,49.4$ \\
\hline \multicolumn{3}{|l|}{ Atypicals vs drug free } \\
\hline \multicolumn{3}{|l|}{ Grey matter excesses in atypicals } \\
\hline (I) $L$ and $R$ thalamus & 310 & $-2.9,-25.8,4.8$ \\
\hline \multicolumn{3}{|l|}{ Typicals vs atypicals } \\
\hline \multicolumn{3}{|l|}{ Grey matter deficits in typicals } \\
\hline (I) L Middle temporal gyrus (BA 2I) & 196 & $-53.0,-14.6,-10.4$ \\
\hline
\end{tabular}

$\mathrm{R}=$ right; $\mathrm{L}=$ left; $\mathrm{BA}=$ Broadmann's area. the nine subjects taking other atypical antipsychotics were removed from this correlational analysis).

Subjects on typical antipsychotics in comparison with subjects on atypical antipsychotics. In comparison with subjects on atypical antipsychotics, subjects taking typical antipsychotics had a cluster of grey matter deficit localized on the left middle temporal gyrus $(P=0.002)$ (Table 2 , Figure 1c).

\section{DISCUSSION}

Our study explored brain structural changes associated with the use of typical or atypical antipsychotics in an epidemiologically based sample of patients at their first psychotic episode. Our results suggest that, in comparison with a drug-free group, treatment with typical antipsychotics may be associated with brain changes in both basal ganglia and cortical areas (lobulus paracentralis, anterior cingulate gyrus, superior and medial frontal gyri, superior and middle temporal gyri, insula, and precuneus), while treatment with atypical antipsychotics may be associated with enlargement of the thalami. However, the limited differences in the direct comparison between typical and atypical groups suggest that the two classes of antipsychotics may share some common effects on brain structure, and that the differences between the effects of typical and atypical drugs may be mostly quantitative, rather than qualitative; therefore, these effects would only appear as significant when each type of antipsychotic is compared to a drug-free group. Of all these findings, only the reduction in the temporal areas seems to be distinctively associated with treatment with typicals. To our knowledge, this is a unique structural MRI study of first episode psychoses, which has explored the effect of different antipsychotics on the whole brain using a voxel-based analysis.

\section{Methodological Issues}

Our study has three major strengths. First, it was conducted on a epidemiologically based catchment-area first episode psychosis sample; this reduced the chances of selection bias, as patients were recruited in chronological order of presentation (although the significance of this strength might have been reduced by the fact that the samples sizes were relatively small, and by the fact that unknown factors may have affected the willingness of our subjects to undergo MRI scanning). Second, as patients were at their first psychotic episode, they had been treated for a relatively short time, and collecting a complete life-antipsychotic medication history was not difficult. Third, we used voxelbased analysis, which not only permits evaluation of the entire brain, but it is also automated and therefore free of the intra/interoperator reliabilities problems associated with manual tracings. The statistical validity of the voxel-based method we used has been extensively tested and the validation process has been described in detail (Bullmore et al, 1999; Suckling et al, 1999a, b). Although there has been some debate on voxel-based methodology (Bookstein, 2001), studies using this approach, including the method we used here, have produced consistent results in the 
a

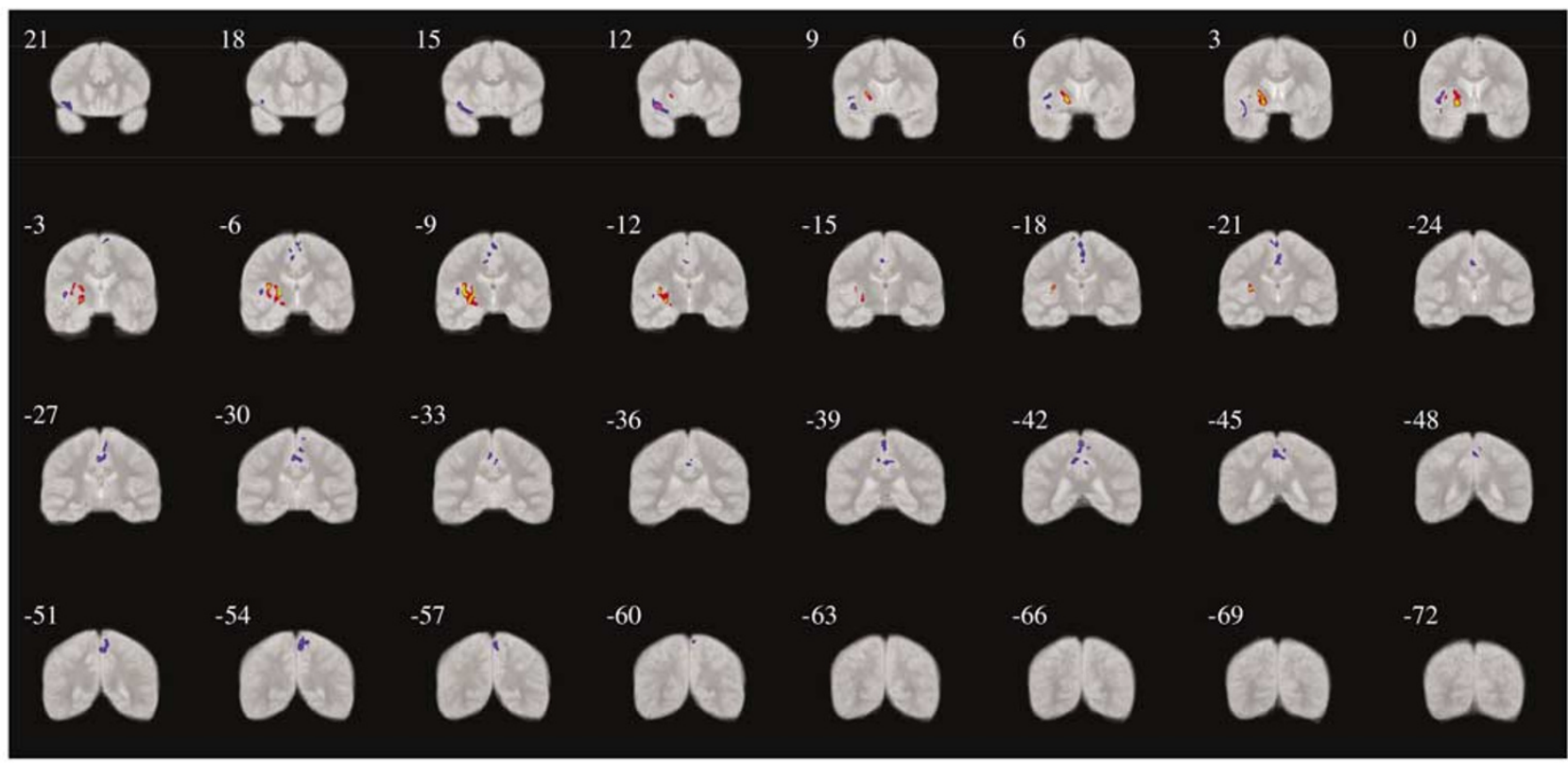

b

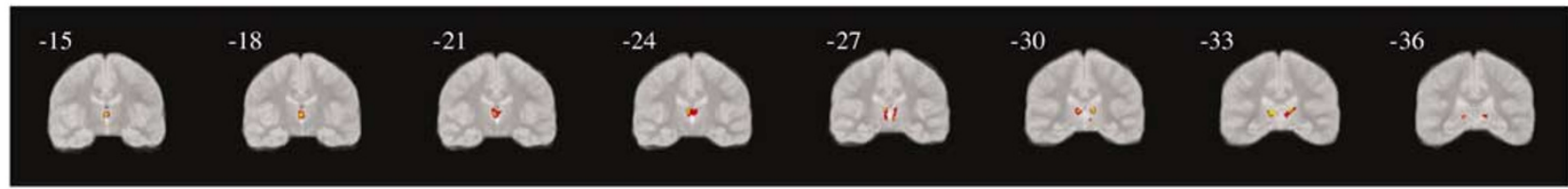

C

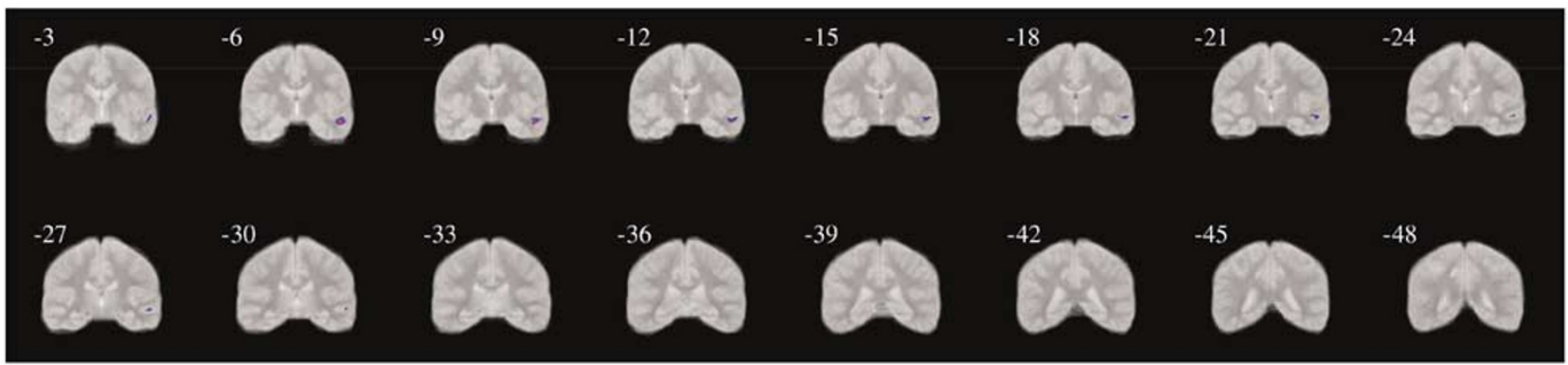

Figure I (a) Subjects on typical antipsychotics vs drug-free subjects. Regions of deficits (blue) and excesses (red) for grey matter in subjects taking typicals. The left side of the image corresponds to the right side of the brain. Numbers refer to the approximate $y$ coordinates in the standard space of Talairach and Tournoux. (b) Subjects on atypical antipsychotics vs drug-free subjects. Regions of excesses (red) for grey matter in subjects taking atypicals. The left side of the image corresponds to the right side of the brain. Numbers refer to the approximate $y$ coordinates in the standard space of Talairach and Tournoux. (c) Subjects on typical antipsychotics vs subjects on atypical antipsychotics. Regions of deficits (blue) for grey matter in subjects taking typicals. The left side of the image corresponds to the right side of the brain. Numbers refer to the approximate $y$ coordinates in the standard space of Talairach and Tournoux.

evaluation of patients with psychosis (Ashburner and Friston, 2000; Job et al, 2002, 2003; Pantelis et al, 2003; Shapleske et al, 2002; Sigmundsson et al, 2001; Watkins et al, 2002). Of course, VBM analyses are exploratory in nature, and we believe that the results obtained in this study need to be corroborated by future hypothesis-driven studies.

The main limitation of this study is its nonrandomized design. As our study was conducted in intact groups, we want to emphasize that baseline differences between the three groups may have affected the findings. For example, as the decision to treat with a specific antipsychotic, or indeed not to treat, was clinically based, some illness features may have influenced the treatment decision. The brain differences observed could reflect different patholo- gical processes underlying different types of psychosis in the three groups, rather than the effect of different treatments. For example, the fact that schizophrenia occurred more often in the atypical antipsychotic group may reflect a bias to use atypical antipsychotics in those patients whose psychotic symptoms indicate a diagnosis of schizophrenia. Also, the premorbid IQ scores of the atypical antipsychotic group were significantly lower than the other two groups, suggesting that perhaps patients with worse cognitive function were more likely to be prescribed atypical antipsychotics. It is possible that those patients who could be maintained drug-free were more likely to have symptoms that were tolerable, less disruptive or debilitating, and that these were associated with a difference in brain 
structure. Thus, the differences we observed might have been due to the pathophysiology of psychosis and its associated cognitive deficits, rather than to the effects of typical or atypical antipsychotic medications per se. However, we feel that our results support the notion that the brain changes described are linked, at least in part, to the different antipsychotic treatments. In fact, most of the clinical differences are present between patients on atypicals and drug-free patients, but subjects on atypicals only show enlargement of the thalami in comparison to the drug-free. Most, if not all, the differences we observed are between the group on typicals and the drug-free, which are similar in terms of diagnosis and cognitive function. Moreover, when we controlled for the effect of factors that differed across the groups, such as premorbid IQ and years of education, the results were not affected. Finally, the groups did not significantly differ in the profile of symptom domains, and this makes it unlikely that the structural brain abnormalities associated with each group could be due to differences in distinct clinical symptom domains associated with specific neurocircuits.

Finally, we chose not to use a group of healthy controls, because comparing patients and controls would introduce an important potential confounder: the fact that patients suffer from psychosis, while the controls do not. This kind of comparison could inform us on the differences in brain structure between groups of subjects with a psychosis and a healthy population. On the other hand, it would make it difficult to distinguish which brain changes are related to the use of a particular antipsychotic and which are related to psychosis. Our approach has been used previously in a number of studies that have looked at the effect of antipsychotics on brain structure (Braus et al, 2001, 2002; Corson et al, 1999; Miller et al, 1997a). However, in order to establish whether our three patient groups were representative of a first episode psychosis population, we have run a parallel series of VBM comparisons of each of these three patient groups with matched healthy controls (unpublished data). In comparison to controls, all three patient groups showed a reduction of grey matter in a reas previously reported as affected in schizophrenia (frontal lobe, superior temporal gyrus, insula), together with increase of the basal ganglia volume (and in the case of the atypicals, also of the thalamus). We are therefore confident that our patient group is representative of first episode psychosis patients.

\section{Proposed Effects of Typical and Atypical Antipsychotics on Brain Structure}

We have found that subjects taking typicals have significantly larger basal ganglia volume than drug-free subjects. This finding is in accordance with a large number of studies that have reported an increase in size and blood flow of these subcortical structures in patients taking typical antipsychotics (Chakos et al, 1994; DeLisi et al, 1991; Gur et al, 1998b; Miller et al, 2001). This volume increase can be reverted by suspension of the drug or switching to clozapine, an atypical antipsychotic (Chakos et al, 1995; Keshavan et al, 1994). It remains unclear what mechanisms underlie the basal ganglia increase. Structural MRI studies may highlight a change in brain volume, but any explana- tion of the pathophysiological mechanisms underlying these volume changes can only be speculative. In fact, what is detected as a change in volume could be for example the consequence of changes in tissue perfusion, fat, or water content (Weinberger and McClure, 2002). This is particularly important when using voxel-based methods of image analysis, and when studying the effect of pharmacological treatment, as different duration of exposure may induce different changes. We believe that these issues need to be taken into account when interpreting our findings. Still, some mechanisms have tentatively been put forward in the literature to try and explain the increase in basal ganglia volume in association with typical antipsychotics use. For example, typical antipsychotics have an antagonistic effect on D2 receptors that could explain changes in structures rich in these receptors, such as the basal ganglia. The chronic block of D2 may lead to a proliferation of D2 receptors, and the resulting increased metabolism and blood flow may lead to an increase in size of the ganglia (Miller et al, 1997a). It is also possible that this D2 proliferation induces changes in the dendritic tree that then lead to an increase in volume (Miller et al, 1997a). Indeed, atypical drugs also bind to D2, and the lack of differences in basal ganglia volume between subjects on typicals and subjects on atypicals suggest that basal ganglia may also be enlarged in association with atypicals use, although the effect may be less marked.

Interestingly, subjects taking atypical antipsychotics showed a significant enlargement of another subcortical area, the thalamus, when compared with drug-free subjects. This finding is similar to a previous report, using a region of interest technique, of an enlargement of thalamic volume associated with use of atypical antipsychotics (Gur et al, 1998b; Miller et al, 2001). It also supports the description of thalamic enlargement in association with symptoms improvement following treatment with atypical antipsychotics (Strungas et al, 2003). The thalamus is part of brain circuits that modulate perception, emotion, and thinking (Crosson and Hughes, 1987), and its volume seems to be reduced in patients with schizophrenia (Andreasen et al, 1994). Owing to its role in integrating brain functions, the thalamus could be involved in mediating the clinical effects of antipsychotic drugs, and it has been indicated as one of the sites of action of both typical and atypical antipsychotic drugs (Cohen et al, 1998, 2003). Again, the fact that the thalamus volumes were not different between typicals and atypicals groups would support that both classes of drugs may affect this region, but to a different extent. For example, differences between the effects of typical and atypical drugs have been described in thalamic GABA-mediated neural transmission (Sakai et al, 2001), monoamine metabolism (Kikumoto et al, 1993), in thalamic cerebral blood volume (Cohen and Yurgelun-Todd, 2001), and in the expression of the protein Fos, which is a marker of cellular activation (Cohen et al, 2003). It is possible that the limbic selectivity of D2/D3 receptor occupancy of atypical antipsychotics is reflected on different effects on thalamus.

We found that typical antipsychotics are associated with a reduction of volume of frontal areas (paracentral lobule, anterior cingulate, superior and medial frontal gyri), 
temporal-insular areas, and precuneus. A volume reduction of frontal lobe has been described by Gur et al (1998a) in a region of interest study, in correlation with higher typical antipsychotic dose. To our knowledge, ours is the first report to indicate regional reductions in the frontal lobe in first episode psychotic patients taking typical antipsychotics in comparison with drug-free subjects. These cortical changes are difficult to interpret and again they might be interpreted as reflecting differences in tissue hydration or metabolism. In fact, use of haloperidol (a typical antipsychotic) has been associated with decreased frontal cerebral blood flow (possibly bringing a reduction in size) in comparison to risperidone (Bartlett et al, 1991; Miller et al, 2001). Different effects of typical and atypical antipsychotics on $\mathrm{N}$-acetylaspartate (NAA) signal (a measure of neuronal viability) in frontal areas has been reported by spectroscopy studies, with typicals being associated with NAA signal reduction in comparison to atypicals (Ende et al, 2000; Heimberg et al, 1998). An increase in functional activation of the frontal lobe following substitution of a typical with an atypical antipsychotic has also been reported using fMRI (Honey et al, 1999). Once again, the lack of difference between subjects on typicals and subject on atypicals may be interpreted as showing some, albeit smaller, effects of atypicals on these structures.

In our study, a reduction in temporal areas seems to be specifically associated with treatment with typical antipsychotic, as it is present in comparison with both the drug-free and the atypicals group. The temporal lobe has been identified as a site rich in extrastriatal cortical D2 receptors, which may have pathophysiological significance for schizophrenia and therefore be possible sites of action for antipsychotics (Okubo et al, 1999). Our finding of a grey matter deficit in the insula may be the structural manifestation of insular hypometabolism in subjects on treatment or previously treated with haloperidol (Desco et al, 2003; Kim et al, 2000). Also, insular activity has been reported as improved following switch from haloperidol to risperidone (Molina et al, 2003). However, other studies on the effect of haloperidol did not show any selective decrease of insular activity (Bartlett et al, 1994; Holcomb et al, 1996).

Finally, we observed a reduction of the cortex of the precuneus, again predominantly associated with typicals. This is an area involved with attention, imagery, and memory processes, and it has been reported as reduced in volume (Shapleske et al, 2002) and altered in function (Kumari et al, 2002; Shapleske et al, 2002) in schizophrenia. The precuneus may be involved in the psychopathology of schizophrenia, and as such could be a potential site of action for antipsychotics.

\section{Conclusion}

On the basis of our findings, we would like to suggest that, even after short-term treatment, both typical and atypical antipsychotics affect brain structure. Although these two classes of drugs may share some common effects on brain structure, there seem to be important quantitative differences in the extent of these effects, which may explain some of the differences in their clinical profile and side effects. The hypotheses proposed in this paper will need to be tested in future ad hoc studies using a randomized design.

\section{ACKNOWLEDGEMENTS}

The ÆSOP study was funded by the Medical Research Council (United Kingdom). P Dazzan holds a NARSAD Young Investigator Award. We thank the Stanley Medical Research Foundation for their support. We wish to thank the ÆSOP researchers, who helped with the data collection and the patients who took part to the study.

\section{REFERENCES}

Andreasen NC, Arndt S, Swayze V, Cizadlo T, Flaum M, O’Leary D et al (1994). Thalamic abnormalities in schizophrenia visualized through magnetic resonance image averaging. Science 266: 294-298.

Annett M (1970). A classification of hand preference by association analysis. Br J Psychol 61: 303-321.

Ashburner J, Friston KJ (2000). Voxel-based morphometry - the methods. Neuroimage 11: 805-821.

Bartlett EJ, Brodie JD, Simkowitz P, Dewey SL, Rusinek H, Wolf AP et al (1994). Effects of haloperidol challenge on regional cerebral glucose utilization in normal human subjects. Am J Psychiatry 151: 681-686.

Bartlett EJ, Wolkin A, Brodie JD, Laska EM, Wolf AP, Sanfilipo M (1991). Importance of pharmacologic control in PET studies: effects of thiothixene and haloperidol on cerebral glucose utilization in chronic schizophrenia. Psychiatry Res 40: 115-124.

Bazire S (1998). Psychotropic Drug Directory: The Professionals' Pocket Handbook and Aide Memoire. Quay Books: Salisbury.

Bezchlibnyk-Butler K, Jeffries J (2000). Clinical Handbook of Psychotropic Drugs, 10th edn Hogrefe \& Huber Publishers: Seattle.

Bookstein FL (2001). 'Voxel-based morphometry' should not be used with imperfectly registered images. Neuroimage 14: 1454-1462.

Braus DF, Ende G, Weber-Fahr W, Demirakca T, Henn FA (2001). Favorable effect on neuronal viability in the anterior cingulate gyrus due to long-term treatment with atypical antipsychotics: an MRSI study. Pharmacopsychiatry 34: 251-253.

Braus DF, Ende G, Weber-Fahr W, Demirakca T, Tost H, Henn FA (2002). Functioning and neuronal viability of the anterior cingulate neurons following antipsychotic treatment: MRspectroscopic imaging in chronic schizophrenia. Eur Neuropsychopharmacol 12: 145-152.

Bullmore ET, Suckling J, Overmeyer S, Rabe-Hesketh S, Taylor E, Brammer MJ (1999). Global, voxel, and cluster tests, by theory and permutation, for a difference between two groups of structural MR images of the brain. IEEE Trans Med Imaging 18: $32-42$.

Carlsson A (1978). Antipsychotic drugs, neurotransmitters, and schizophrenia. Am J Psychiatry 135: 165-173.

Chakos MH, Lieberman JA, Alvir J, Bilder R, Ashtari M (1995). Caudate nuclei volumes in schizophrenic patients treated with typical antipsychotics or clozapine. Lancet 345: 456-457.

Chakos MH, Lieberman JA, Bilder RM, Borenstein M, Lerner G, Bogerts B et al (1994). Increase in caudate nuclei volumes of first-episode schizophrenic patients taking antipsychotic drugs. Am J Psychiatry 151: 1430-1436.

Christensen J, Holcomb J, Garver DL (2004). State-related changes in cerebral white matter may underlie psychosis exacerbation. Psychiatry Res 130: 71-78.

Cohen BM, Cherkerzian S, Ma J, Ye N, Wager C, Lange N (2003). Cells in midline thalamus, central amygdala, and nucleus 
accumbens responding specifically to antipsychotic drugs. Psychopharmacology (Berlin) 167: 403-410.

Cohen BM, Wan W, Froimowitz MP, Ennulat DJ, Cherkerzian S, Konieczna H (1998). Activation of midline thalamic nuclei by antipsychotic drugs. Psychopharmacology (Berlin) 135: 37-43.

Cohen BM, Yurgelun-Todd D (2001). Alterations of thalamic activity in schizophrenia and in response to antipsychotic drugs: studies in the legacy of Seymour S Kety. Neuropsychopharmacology 25: 305-312.

Corson PW, Nopoulos P, Miller DD, Arndt S, Andreasen NC (1999). Change in basal ganglia volume over 2 years in patients with schizophrenia: typical versus atypical neuroleptics. Am J Psychiatry 156: 1200-1204.

Crosson B, Hughes CW (1987). Role of the thalamus in language: is it related to schizophrenic thought disorder? Schizophr Bull 13: 605-621.

Dazzan P, Morgan KD, Orr KG, Hutchinson G, Chitnis X, Suckling $\mathrm{J}$ et al (2004). The structural brain correlates of neurological soft signs in AESOP first-episode psychoses study. Brain 127: 143-153.

Dazzan P, Murray RM (1999). Schizophrenia is (not simply) a neurodevelopmental disorder. Epidemiol Psichiatr Soc 8: 235-241.

Dazzan P, Murray RM (2002). Neurological soft signs in firstepisode psychosis: a systematic review. Br J Psychiatry Suppl 43: s50-s57.

DeLisi LE, Hoff AL, Schwartz JE, Shields GW, Halthore SN, Gupta SM et al (1991). Brain morphology in first-episode schizophrenic-like psychotic patients: a quantitative magnetic resonance imaging study. Biol Psychiatry 29: 159175 .

Desco M, Gispert JD, Reig S, Sanz J, Pascau J, Sarramea F et al (2003). Cerebral metabolic patterns in chronic and recent-onset schizophrenia. Psychiatry Res 122: 125-135.

Ende G, Braus DF, Walter S, Weber-Fahr W, Soher B, Maudsley AA et al (2000). Effects of age, medication, and illness duration on the $\mathrm{N}$-acetyl aspartate signal of the anterior cingulate region in schizophrenia. Schizophr Res 41: 389-395.

Farde L, Hall H, Ehrin E, Sedvall G (1986). Quantitative analysis of D2 dopamine receptor binding in the living human brain by PET. Science 231: 258-261.

Grunder G, Vernaleken I, Muller MJ, Davids E, Heydari N, Buchholz HG et al (2003). Subchronic haloperidol downregulates dopamine synthesis capacity in the brain of schizophrenic patients in vivo. Neuropsychopharmacology 28: 787-794.

Gur RE, Cowell P, Turetsky BI, Gallacher F, Cannon T, Bilker W et al (1998a). A follow-up magnetic resonance imaging study of schizophrenia. Relationship of neuroanatomical changes to clinical and neurobehavioral measures. Arch Gen Psychiatry 55: 145-152.

Gur RE, Maany V, Mozley PD, Swanson C, Bilker W, Gur RC (1998b). Subcortical MRI volumes in neuroleptic-naive and treated patients with schizophrenia. Am J Psychiatry 155: 1711-1717.

Heimberg C, Komoroski RA, Lawson WB, Cardwell D, Karson CN (1998). Regional proton magnetic resonance spectroscopy in schizophrenia and exploration of drug effect. Psychiatry Res 83: 105-115.

Holcomb HH, Cascella NG, Thaker GK, Medoff DR, Dannals RF, Tamminga CA (1996). Functional sites of neuroleptic drug action in the human brain: PET/FDG studies with and without haloperidol. Am J Psychiatry 153: 41-49.

Honey GD, Bullmore ET, Soni W, Varatheesan M, Williams SC, Sharma T (1999). Differences in frontal cortical activation by a working memory task after substitution of risperidone for typical antipsychotic drugs in patients with schizophrenia. Proc Natl Acad Sci USA 96: 13432-13437.
Huang YH, Tsai SJ, Huang HJ, Sim CB (1999). The effect of acute administration of risperidone on local cerebral glucose utilization in the rat. Eur J Pharmacol 370: 257-261.

Job DE, Whalley HC, McConnell S, Glabus M, Johnstone EC, Lawrie SM (2002). Structural gray matter differences between first-episode schizophrenics and normal controls using voxelbased morphometry. Neuroimage 17: 880-889.

Job DE, Whalley HC, McConnell S, Glabus M, Johnstone EC, Lawrie SM (2003). Voxel-based morphometry of grey matter densities in subjects at high risk of schizophrenia. Schizophr Res 64: 1-13.

Keshavan MS, Bagwell WW, Haas GL, Sweeney JA, Schooler NR, Pettegrew JW (1994). Changes in caudate volume with neuroleptic treatment. Lancet 344: 1434.

Kikumoto O, Okamoto Y, Hayakawa H, Yokota N, Kawai K, Motohashi $\mathrm{N}$ et al (1993). Effects of risperidone on catalepsy and cerebral dopamine, serotonin and GABA metabolism in the rat: comparison with haloperidol. Jpn J Psychopharmacol 13: $39-42$.

Kim JJ, Mohamed S, Andreasen NC, O’Leary DS, Watkins GL, Boles Ponto LL et al (2000). Regional neural dysfunctions in chronic schizophrenia studied with positron emission tomography. Am J Psychiatry 157: 542-548.

Kumari V, Gray JA, Honey GD, Soni W, Bullmore ET, Williams SC et al (2002). Procedural learning in schizophrenia: a functional magnetic resonance imaging investigation. Schizophr Res 57: 97-107.

Meltzer HY, Matsubara S, Lee JC (1989). Classification of typical and atypical antipsychotic drugs on the basis of dopamine D-1, D-2 and serotonin2 pKi values. J Pharmacol Exp Ther 251: 238-246.

Meltzer HY, Park S, Kessler R (1999). Cognition, schizophrenia, and the atypical antipsychotic drugs. Proc Natl Acad Sci USA 96: 13591-13593.

Miller DD, Andreasen NC, O’Leary DS, Rezai K, Watkins GL, Ponto LL et al (1997a). Effect of antipsychotics on regional cerebral blood flow measured with positron emission tomography. Neuropsychopharmacology 17: 230-240.

Miller DD, Andreasen NC, O'Leary DS, Watkins GL, Boles Ponto LL, Hichwa RD (2001). Comparison of the effects of risperidone and haloperidol on regional cerebral blood flow in schizophrenia. Biol Psychiatry 49: 704-715.

Miller DD, Rezai K, Alliger R, Andreasen NC (1997b). The effect of antipsychotic medication on relative cerebral blood perfusion in schizophrenia: assessment with technetium-99m hexamethylpropyleneamine oxime single photon emission computed tomography. Biol Psychiatry 41: 550-559.

Molina V, Gispert JD, Reig S, Sanz J, Pascau J, Santos A et al (2003). Cerebral metabolism and risperidone treatment in schizophrenia. Schizophr Res 60: 1-7.

Nelson HE, Willison JR (1991). National Adult Reading Test (Part II) Test Manual. NFER-NELSON: Windsor.

Okubo Y, Olsson $\mathrm{H}$, Ito $\mathrm{H}$, Lofti $\mathrm{M}$, Suhara T, Halldin $\mathrm{C}$ et al (1999). PET mapping of extrastriatal D2-like dopamine receptors in the human brain using an anatomic standardization technique and [11C]FLB 457. Neuroimage 10: 666-674.

Pantelis C, Velakoulis D, McGorry PD, Wood SJ, Suckling J, Phillips LJ et al (2003). Neuroanatomical abnormalities before and after onset of psychosis: a cross-sectional and longitudinal MRI comparison. Lancet 361: 281-288.

Sakai K, Gao XM, Hashimoto T, Tamminga CA (2001). Traditional and new antipsychotic drugs differentially alter neurotransmission markers in basal ganglia-thalamocortical neural pathways. Synapse 39: 152-160.

Shapleske J, Rossell SL, Chitnis XA, Suckling J, Simmons A, Bullmore ET et al (2002). A computational morphometric MRI study of schizophrenia: effects of hallucinations. Cereb Cortex 12: $1331-1341$. 
Sigmundsson T, Suckling J, Maier M, Williams S, Bullmore E, Greenwood $\mathrm{K}$ et al (2001). Structural abnormalities in frontal, temporal, and limbic regions and interconnecting white matter tracts in schizophrenic patients with prominent negative symptoms. Am J Psychiatry 158: 234-243.

Strungas S, Christensen JD, Holcomb JM, Garver DL (2003). Staterelated thalamic changes during antipsychotic treatment in schizophrenia: preliminary observations. Psychiatry Res 124: 121-124.

Suckling J, Brammer MJ, Lingford-Hughes A, Bullmore ET (1999a). Removal of extracerebral tissues in dual-echo magnetic resonance images via linear scale-space features. Magn Reson Imaging 17: 247-256.

Suckling J, Sigmundsson T, Greenwood K, Bullmore ET (1999b). A modified fuzzy clustering algorithm for operator independent brain tissue classification of dual echo MR images. Magn Reson Imaging 17: 1065-1076.

Talairach J, Tournoux P (1988). Co-planar Stereotaxic Atlas of the Human Brain. Thieme Medical Publisher: New York.

Taylor D, McConnell D, McConnell H, Abel K, Kerwin R (1999). The Bethlem and Maudsley NHS Trust Prescribing Guidelines. Martin Dunitz: London.
Watkins KE, Vargha-Khadem F, Ashburner J, Passingham RE, Connelly A, Friston KJ et al (2002). MRI analysis of an inherited speech and language disorder: structural brain abnormalities. Brain 125: 465-478.

Weinberger DR, McClure RK (2002). Neurotoxicity, neuroplasticity, and magnetic resonance imaging morphometry: what is happening in the schizophrenic brain? Arch Gen Psychiatry 59: 553-558.

Wing JK, Cooper JE, Sartorius N (1974). The Measurement and Classification of Psychiatric Symptoms. Cambridge University Press: Cambridge.

Wing JK, Sturt E (1978). The PSE-ID-CATEGO System: Supplementary Manual. Medical Research Council: London.

World Health Organisation (1992). The ICD-10 Classification of Mental and Behavioural Disorders: Clinical Descriptions and Diagnostic Guidelines. World Health Organisation: Geneva.

World Health Organisation (1994). Schedules for Clinical Assessment in Neuropsychiatry (SCAN) - Version 2.0. World Health Organisation: Geneva.

Wotanis J, Hanak SE, Wettstein JG, Black MD (2003). Comparative analysis of acute and chronic administration of haloperidol and clozapine using [3H] 2-deoxyglucose metabolic mapping. Schizophr Res 61: 195-205. 